\title{
Effect of InGaAs capping layer on the properties of InAs/InGaAs quantum dots and lasers
}

\author{
F. Y. Chang, C. C. Wu, and H. H. Lin a) \\ Department of Electrical Engineering and Graduate Institute of Electronics Engineering, National Taiwan \\ University, Taipei, Taiwan, Republic of China
}

(Received 20 January 2003; accepted 18 April 2003)

\begin{abstract}
We report the effects of $\operatorname{In}_{0.33} \mathrm{Ga}_{0.67}$ As capping layers on the structural and optical properties of InAs self-organized quantum dots grown by gas-source molecular-beam epitaxy. With different deposition methods for the InGaAs capping layer, the quantum-dot density can be adjusted from $2.3 \times 10^{10}$ to $1.7 \times 10^{11} \mathrm{~cm}^{-2}$. As-cleaved 3.98-mm-long diode laser using triple stacks of InAs quantum dots with the capping layer grown by GaAs/InAs sequential binary growth demonstrates an emission wavelength of $1305 \mathrm{~nm}$ and a threshold current density of $360 \mathrm{~A} / \mathrm{cm}^{2}$. A ground-state saturation gain of $16.6 \mathrm{~cm}^{-1}$ is achieved due to the high dot density. (C) 2003 American Institute of Physics. [DOI: 10.1063/1.1585125]
\end{abstract}

For almost a decade, self-assembled $\operatorname{In}(\mathrm{Ga})$ As quantum dots (QDs) formed by Stranski-Krastanow growth mode have attracted much attention because of their great potential for optoelectronic applications. The QD laser, one of its important applications, is expected to have excellent performances due to the delta-function-like density of states in the dots. In the past few years, a lot of significant improvements on the performance of QD lasers have been reported, including low threshold current density, ${ }^{1}$ room temperature continuous-wave (cw) operation, ${ }^{2}$ and high characteristic temperature $\left(T_{0}\right){ }^{3}$ Room temperature ground-state lasing at $1.3 \mu \mathrm{m}$ has been achieved in lasers with $\operatorname{In}(\mathrm{Ga}) \mathrm{As}$ QD medium grown by molecular-beam epitaxy (MBE) on GaAs substrates using submonolayer (ML) deposition ${ }^{4}$ and InGaAs capping layers. ${ }^{5}$ Although the InGaAs QDs grown by sub-ML deposition method can extend the emission wavelength to $1.3 \mu \mathrm{m}$, the relatively low dot density (about $1-2 \times 10^{10} / \mathrm{cm}^{2}$ ) limits the maximal gain of the ground state. Another approach for long wavelength QD lasers, which has been reported intensively, is associated with either covering or embedding InAs QDs with InGaAs layers. ${ }^{5,6}$ It has been shown that the InGaAs layers can provide several advantages, such as extending the emission wavelength to $1.3 \mu \mathrm{m}$, narrowing the photoluminescence (PL) linewidths, ${ }^{7}$ and increasing the dot density. ${ }^{8-10}$ In general, the reported InGaAs buffer or capping layers are with an In mole fraction ranging from 0.09 to $0.15 .^{5-10}$ In this work, $\operatorname{In}_{0.33} \mathrm{Ga}_{0.67}$ As with a much higher In content is adopted for the capping layer overgrown on InAs QDs. Furthermore, three types of InAs/ InGaAs QDs, InAs covered by $\mathrm{Ga} / \mathrm{InAs}$ supply (type-A QDs), InAs covered by GaAs/InAs sequential binary growth (type-B QDs), and InAs and InGaAs with usual MBE growth (type-C QDs), are studied to optimize the optical properties of the QDs. The dot density ranging from $2.3 \times 10^{10}$ to 1.7 $\times 10^{11} \mathrm{~cm}^{-2}$ can be achieved without a great PL intensity degradation through these deposition methods. With InAs

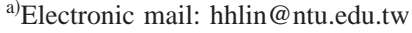

QDs covered by GaAs/InAs sequential binary growth, we have successfully achieved $1.3 \mu \mathrm{m}$ lasing at room temperature with good device quality.

In this work, the QDs and lasers were grown on $\mathrm{Si}$ doped (100) GaAs substrates by gas-source MBE. The laser structure consists of a 500-nm-thick $n$-type GaAs buffer layer, a $1.7-\mu \mathrm{m}$-thick $n$-type $\mathrm{In}_{0.49} \mathrm{Ga}_{0.51} \mathrm{P}$ lower cladding layer, a 200-nm-thick GaAs waveguide layer in which the QD active medium is embedded, a 1.6- $\mu$ m-thick $p$-type $\mathrm{In}_{0.49} \mathrm{Ga}_{0.51} \mathrm{P}$ upper cladding layer, and a 200-nm-thick heavily Be-doped GaAs contact layer. In the center of the waveguide are QD layers. In this study, three kinds of QDs, type-A QDs, type-B QDs, and type-C QDs, were grown. For growing each QD layer, 2.0 ML InAs was first deposited and followed by a $9.0 \mathrm{ML} \mathrm{In}_{0.33} \mathrm{Ga}_{0.67}$ As capping layer. In type-A QDs, the InGaAs layer was grown in the sequence of the following steps: $0.5 \mathrm{ML}$ Ga deposition, $5 \mathrm{~s} \mathrm{As}_{2}$ illumination, and $0.25 \mathrm{ML}$ InAs deposition. Between the steps, there is a $2.5 \mathrm{~s}$ interruption without $\mathrm{As}_{2}$ protection. The cycle was repeated 12 times. In type-B QDs, the cycle includes $0.5 \mathrm{ML}$ GaAs followed by $10 \mathrm{~s}$ interruption and 0.25 ML InAs followed by $2.5 \mathrm{~s}$ interruption. During the interruption, the sample was protected by $\mathrm{As}_{2}$ beam, and the cycle was also repeated 12 times. In type-C QDs, all of $\mathrm{In}, \mathrm{Ga}$, and $\mathrm{As}_{2}$ beams were used at the same time during the deposition of the InGaAs layer. Between the QD layers are 25-nm-thick GaAs spacers. The growth temperatures of InAs QDs and InGaAs capping layers were both $485^{\circ} \mathrm{C}$. After the epitaxial growth, the samples were processed into $50-\mu \mathrm{m}$-wide broad area lasers with different cavity lengths. The fabricated lasers were tested under pulsed mode with a pulse width of $4 \mu \mathrm{s}$ and a repetition rate of $500 \mathrm{~Hz}$. PL measurement was also used to study the as-grown QD samples.

Figures 1(a)-1(d) show the SEM images of $2.0 \mathrm{ML}$ InAs, type-A, type-B, and type-C QDs, respectively. The dot density of InAs, type-A, type-B, and type-C QDs are 6.9 $\times 10^{10}, 2.3 \times 10^{10}, 7.6 \times 10^{10}$, and $1.7 \times 10^{11} \mathrm{~cm}^{-2}$, and the corresponding base diameters are 20,34, 29, and $27 \mathrm{~nm}$, respectively. Through different deposition methods for InGaAs capping layer, the dot density changes from 2.3 


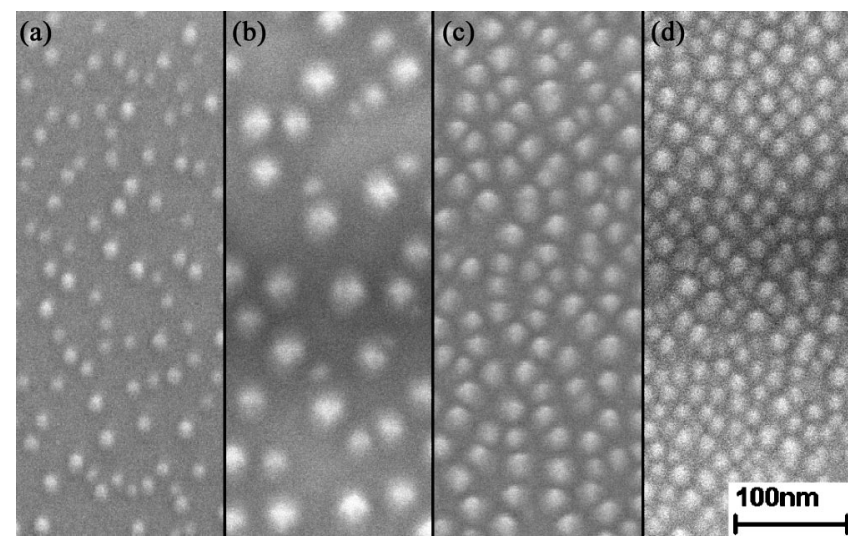

FIG. 1. SEM images of (a) InAs QDs, (b) type-A QDs, (c) type-B QDs, and (d) type-C QDs.

$\times 10^{10}$ to $1.7 \times 10^{11} \mathrm{~cm}^{-2}$. Figure 2(a) shows the room temperature PL spectra of these samples. The PL peak wavelength of InAs, type-A, type-B, and type-C QDs are 1051, 1267, 1318, and $1279 \mathrm{~nm}$, respectively. The corresponding full width at half maximum (FWHM) linewidths are 66, 54, 44 , and $60 \mathrm{meV}$. The structural parameters and measured photoluminescence of these four QD samples are summarized in Table I. The deposition method of the InGaAs capping layer influences both the dot density and the emission wavelength of InAs/InGaAs QDs. This can be explained by the redistribution of In and $\mathrm{Ga}$ atoms during the deposition of the InGaAs capping layer on InAs QDs. ${ }^{11}$ A strain field caused by InAs QDs induces the preferential migration of In atoms to InAs QDs and $\mathrm{Ga}$ atoms from the dots. Furthermore, during the overgrowth of the high-In-content InGaAs, new islands are generated at the nucleation center of the InAs wetting layer. In and $\mathrm{Ga}$ atoms have longer surface migration lengths in type-B QDs as compared with those in type-C QDs. Thus more In atoms can migrate to InAs QDs, and hence type-B QDs have bigger dot size and lower dot density. On the other hand, the MBE method provides shorter migration lengths for the adatoms and thus has higher prob-

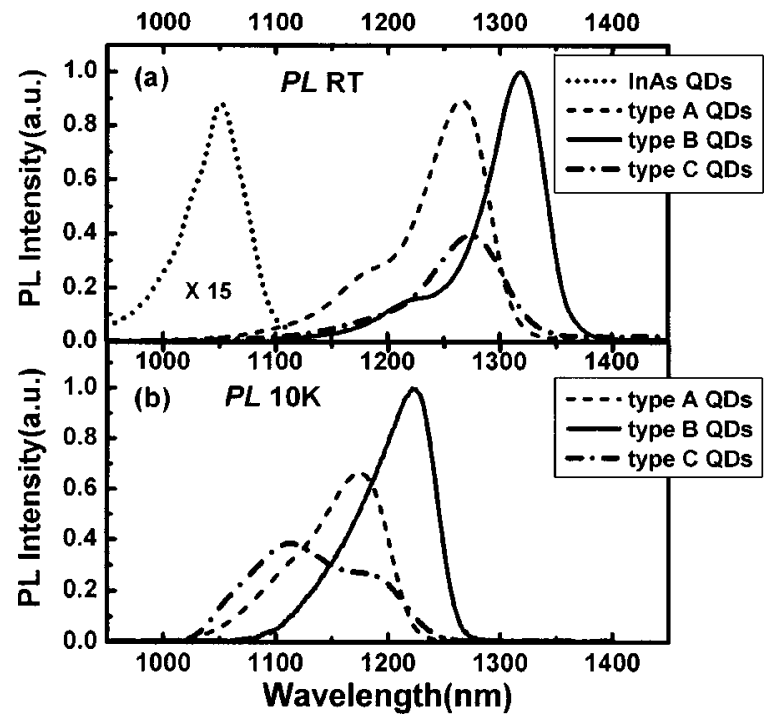

FIG. 2. (a) Room temperature PL spectra of InAs QDs, type-A, type-B, and type-C QDs. (b) PL spectra at $10 \mathrm{~K}$ of type-A, type-B, and type-C QDs. The SEM images of these samples are presented in Fig. 1. SEM images of these samples are presented in Fig. 1. the lasing wavelengths are 1305 and $1197 \mathrm{~nm}$, and the cor-
Downloaded 24 Oct 2003 to 140.112 .103 .13 . Redistribution subject to AlP license or copyright, see http://ojps.aip.org/aplo/aplcr.jsp
TABLE I. Summary of structural parameters and measured photoluminescence of InAs QDs, InAs covered by Ga/InAs supply (type-A QDs), InAs covered by GaAs/InAs sequential binary growth (type-B QDs), and InAs and InGaAs with usual MBE growth (type-C QDs).

\begin{tabular}{lcccc}
\hline \hline QDs & $\begin{array}{c}\text { Dot density } \\
\left(\mathrm{cm}^{-2}\right)\end{array}$ & $\begin{array}{c}\text { Dot diameter } \\
(\mathrm{nm})\end{array}$ & $\begin{array}{c}\text { PL peak } \\
\text { wavelength }(\mathrm{nm})\end{array}$ & $\begin{array}{c}\text { FWHM } \\
(\mathrm{meV})\end{array}$ \\
\hline InAs QDs & $6.9 \times 10^{10}$ & 20 & 1051 & 66 \\
type-A QDs & $2.3 \times 10^{10}$ & 34 & 1267 & 54 \\
type-B QDs & $7.6 \times 10^{10}$ & 29 & 1318 & 44 \\
type-C QDs & $1.7 \times 10^{11}$ & 27 & 1279 & 60 \\
\hline \hline
\end{tabular}

ability of nucleating new islands. Though both the QD size and density increase after capping the high-In-content InGaAs layer in type-B and type-C QDs, the former method gives bigger dot size and significant redshift on the emission wavelength. In type-A QDs, the desorption of In atoms may take place during the interruption stage because of lacking $\mathrm{As}_{2}$ protection. InAs QDs with smaller size could disappear due to the desorption of its In content, and result in even a lower dot density than InAs QDs without InGaAs capping layers. In the deposition of the covering InGaAs layer of type-A QDs, In and As were co-deposited. In fact, interruption of As supply during the In deposition was also tested, and the dot density is even lower. However, the strongest migration ability provided by migration enhanced epitaxy method may greatly enhance the size of the surviving QDs, which is the biggest among the three methods as can be seen in the figures. The vertical exchange of In and $\mathrm{Ga}$ atoms in migration enhanced epitaxy has been ever reported. ${ }^{12}$ According to this theory, the In atoms of the InAs QD and its covering layer may be replaced by Ga atoms, and the reduction of In content in both the dot and covering layer leads to a blueshift in PL transition due to the increment of the band gap energy. Although type-A QDs has the biggest dot size, the reduction in In content dominates the PL characteristics and results in a shorter peak wavelength as compared with type-B and type-C QDs. Figure 2(b) displays the $10 \mathrm{~K}$ PL spectra of type-A, type-B, and type-C QDs. The spectrum of type-C QDs shows two peaks, while type-A and type-B QDs show only one peak. This indicates that there are two groups of QDs coexisting in type-C QDs. This result supports our point that besides the InAs QDs under the InGaAs capping layer, new InGaAs QDs also nucleated on the InAs wetting layer or InGaAs layer in type-C QDs. Although the dot density of type-B QDs is slightly higher than that of InAs QDs, the single peak in their $10 \mathrm{~K}$ PL spectra reveals that most of type-B QDs are InAs/InGaAs QDs. The results in Ref. 11 show that there is a drastic decrease in PL intensity at $1.3 \mu \mathrm{m}$ wavelength region when the In composition of InGaAs capping layer grown by MBE method is over 30\%. However, in our study, we found that the wavelength of type-B QDs can be extended to $1.3 \mu \mathrm{m}$ without optical degradation when In composition of $33 \%$ is used.

The room temperature lasing and PL spectra of the threestack type-B QD lasers are shown in Fig. 3. The PL was measured after the GaAs contact layer and InGaP upper cladding layer were removed. Two PL maxima corresponding to the ground state and first excited state are clearly observed. For as-cleaved lasers with cavity lengths of 3.98 and $0.9 \mathrm{~mm}$, 


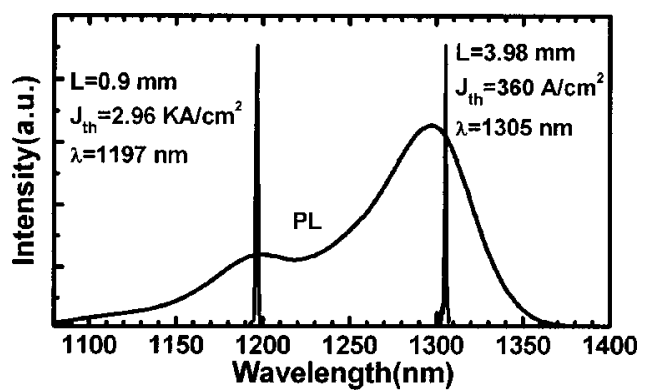

FIG. 3. PL and lasing spectra for InAs/InGaAs QD lasers with type-B QDs.

responding threshold current densities are $360 \mathrm{~A} / \mathrm{cm}^{2}$ and $2.96 \mathrm{kA} / \mathrm{cm}^{2}$. The threshold current density and the lasing wavelength versus reciprocal cavity length plot of type-B QD laser are shown in Fig. 4. The lasing wavelength decreases from 1305 to $1193 \mathrm{~nm}$, and the threshold current density increases from $360 \mathrm{~A} / \mathrm{cm}^{2}$ to $4.84 \mathrm{kA} / \mathrm{cm}^{2}$, as the cavity length is decreased from 3.98 to $0.64 \mathrm{~mm}$. When the cavity length is $0.96 \mathrm{~mm}$, two lasing lines corresponding to the ground state and first excited state transition are observed at the current density of $1.35 J_{\text {th }}$. This reveals the competition between the ground-state and excited-state transitions at the pumping level close to the gain saturation of the ground state. As the cavity length is shorter than $0.96 \mathrm{~mm}$, the lasing line shifts from the ground state to the first excited state, and the threshold current density jumps to $2.96 \mathrm{kA} / \mathrm{cm}^{2}$ abruptly. This can be attributed to the gain saturation of the ground state. As the cavity length is decreased, the mirror loss increases and finally makes the total loss larger than the saturation gain of the ground state. As a result, the device is forced to lase at the first excited state, which has higher order of degeneracy and thus a higher saturation gain and transparency current density. Using linear fit on the plot of inversed external quantum efficiency versus cavity length, the de-

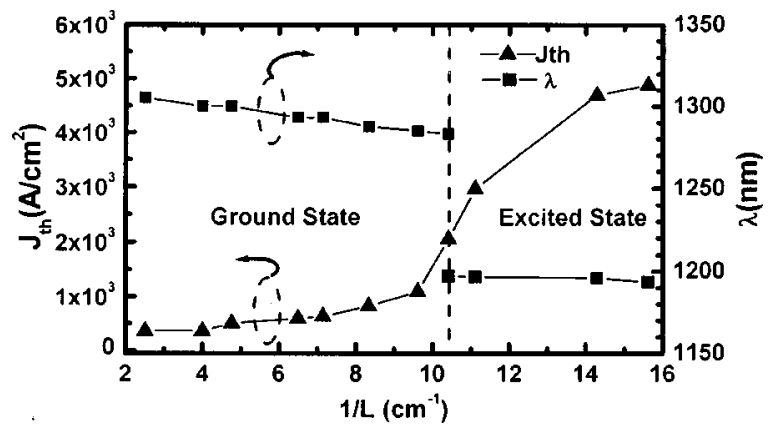

FIG. 4. Threshold current density and lasing wavelength vs reciprocal cavity length plot for type-B QD lasers. duced internal loss $\left(\alpha_{i}\right)$ and internal quantum efficiency $\left(\eta_{i}\right)$ of the ground state in this laser are $4.82 \mathrm{~cm}^{-1}$ and $28.3 \%$, respectively. Using the condition that the gain is equal to the total losses (cavity and mirror) on the laser with 0.96-mmlong cavity, we can find that the saturation gain of the ground state is $16.6 \mathrm{~cm}^{-1}$.

In summary, we have studied the effects of high-Incontent InGaAs capping layer on the structural and optical properties of the QDs. By using different capping methods, the quantum-dot density can be controllably changed from $2.3 \times 10^{10}$ to $1.7 \times 10^{11} \mathrm{~cm}^{-2}$ without a pronounced decrease in PL intensity. QDs with the capping layer grown by GaAs/ InAs sequential binary growth method (type-B QDs) have a dot density as high as $7.6 \times 10^{10} \mathrm{~cm}^{-2}$ and a photoluminescence peak at $1318 \mathrm{~nm}$. We also investigated the characteristics of lasers with type-B QD medium. For an as-cleaved laser with a cavity length of $3.98 \mathrm{~mm}$, the lasing wavelength is $1305 \mathrm{~nm}$ and threshold current density is $360 \mathrm{~A} / \mathrm{cm}^{2}$. By changing the cavity length, the step-like jumps of the lasing wavelength and threshold current density due to the gain saturation of the ground state are observed. The ground-state saturation gain of the type-B QD laser is $16.6 \mathrm{~cm}^{-1}$.

This work was supported by the National Science Council of the Republic of China under Contract No. NSC 912120-E-002-004.

${ }^{1}$ P. G. Eliseev, H. Li, A. Stintz, G. T. Liu, T. C. Newell, K. J. Malloy, and L. F. Lester, Appl. Phys. Lett. 77, 262 (2000).

${ }^{2}$ G. Park, O. B. Shchekin, S. Csutak, D. L. Huffaker, Z. Zou, and D. G. Deppe, Appl. Phys. Lett. 75, 3267 (1999).

${ }^{3}$ M. V. Maximov, I. V. Kochnev, Y. M. Shernyakov, S. V. Zaitsev, N. Y. Gordeev, A. F. Tsatsul'nikov, A. V. Sakharov, I. L. Krestnikov, P. S. Kop'ev, Z. I. Alferov, N. N. Ledentsov, D. Bimberg, A. O. Kosogov, P. Werner, and U. Gosele, Jpn. J. Appl. Phys., Part 1 36, 4221 (1997).

${ }^{4}$ G. Park, D. L. Huffaker, Z. Zou, and D. G. Deppe, IEEE Photonics Technol. Lett. 11, 301 (1999).

${ }^{5}$ V. M. Ustinov, A. E. Zhukov, N. A. Maleev, A. R. Kovsh, S. S. Mikhrin, B. V. Volovik, Yu. G. Musikhin, Yu. M. Shernyakov, M. V. Maximov, A. F. Tsatsul'nikov, N. N. Ledentsov, Zh. I. Alferov, J. A. Lott, and D. Bimberg, J. Cryst. Growth 227-228, 1155 (2001).

${ }^{6}$ A. Stintz, G. T. Liu, H. Li, L. F. Lester, and J. M. Malloy, IEEE Photonics Technol. Lett. 12, 591 (2000).

${ }^{7}$ K. Nishi, H. Saito, S. Sugou, and J. S. Lee, Appl. Phys. Lett. 74, 1111 (1999).

${ }^{8}$ G. Park, O. B. Shchekin, D. L. Huffaker, and D. G. Deppe, IEEE Photonics Technol. Lett. 13, 230 (2000).

${ }^{9}$ A. Stintz, G. T. Liu, A. L. Gray, R. Spillers, S. M. Delgado, and K. J. Malloy, J. Vac. Sci. Technol. B 18, 1496 (2000).

${ }^{10}$ N. T. Yeh, T. E. Nee, J. I. Chyi, C. T. Chia, T. M. Hsu, and C. C. Huang, J. Cryst. Growth 227-228, 1044 (2001).

${ }^{11}$ M. V. Maximov, A. F. Tsatsul'nikov, B. V. Volovik, D. S. Sizov, Yu. M. Shernyakov, I. N. Kaiander, A. E. Zhukov, A. R. Kovsh, S. S. Mikhrin, V. M. Ustinov, and Zh. I. Alferov, Phys. Rev. B 62, 16671 (2000).

${ }^{12}$ H. Yamaguchi and Y. Horikoshi, J. Appl. Phys. 68, 1610 (1990). 